\title{
Coulisses
}

Revue de théâtre

15 | Hiver 1997

Varia

\section{La double naissance scénique du théâtre claudélien : les deux premières mises en scène de L'Annonce faite à Marie}

\section{Alain Beretta}

\section{OpenEdition}

\section{Journals}

Édition électronique

URL : http://journals.openedition.org/coulisses/5057

DOI : 10.4000/coulisses.5057

ISSN : 2546-9460

Éditeur

Presses universitaires de Franche-Comté

\section{Édition imprimée}

Date de publication : 1 janvier 1997

Pagination : 34-40

ISSN : $1150-594 \mathrm{X}$

\section{Référence électronique}

Alain Beretta, «La double naissance scénique du théâtre claudélien : les deux premières mises en scène de L'Annonce faite à Marie », Coulisses [En ligne], 15 | Hiver 1997, mis en ligne le 26 avril 2019, consulté le 22 octobre 2019. URL : http://journals.openedition.org/coulisses/5057 ; DOI : 10.4000/ coulisses. 5057

Ce document a été généré automatiquement le 22 octobre 2019

Coulisses 


\title{
La double naissance scénique du théâtre claudélien : les deux premières mises en scène de L'Annonce faite à Marie ${ }^{1}$
}

\author{
Alain Beretta
}

\section{Une naissance scénique difficile}

1 Le théâtre de Paul Claudel s'est révélé et demeure la forme la plus originale de la production dramatique française dans la première moitié du $\mathrm{XX}^{\mathrm{e}}$ siècle. Mais, comme toute nouveauté géniale, il a longtemps fait peur. Sur le plan littéraire déjà, son écriture à la fois si poétique et si expressive s'est heurtée à une certaine tradition française. Aussi, sur le plan scénique sa réputation et plus encore son succès auprès du grand public furent-ils tardifs : 26 ans séparent la première pièce écrite par Claudel, L'Endormie (1886), et la première pièce jouée, L'Annonce faite à Marie (1912). Pendant tout ce temps, le dramaturge a progressivement évolué des diverses réticences qu'il nourrissait à l'égard de la scène - à la fois pour des raisons personnelles et professionnelles liées à sa carrière diplomatique - vers un désir de plus en plus net d'être enfin joué et reconnu. Mais lorsque, à l'initiative de l'actrice Marie Kalff liée au metteur en scène Lugné-Poe, des projets de représentation ont lieu, en 1909 pour La Jeune Fille Violaine et en 1910 pour Partage de Midi, Claudel doit les refuser, à contrecœur. En juillet 1912, il tente alors sa revanche en suggérant à Lugné-Poe de monter L'Annonce: cette timide proposition allait lancer la fulgurante carrière scénique d'une des pièces les plus jouées du $\mathrm{XX}^{\mathrm{e}}$ siècle dans le monde entier et, à sa suite, de tout le théâtre claudélien.

Pourquoi, en 1912, Claudel propose-t-il précisément L'Annonce? C'est certainement qu'il porte en lui depuis longtemps cette pièce nourrie de ses souvenirs d'enfance, et qu'il n'a cessé de remanier pour développer ses vertus scéniques, des deux versions successives de sa première appellation, La Jeune Fille Violaine (1892 et 1899), jusqu'à son 
titre définitif, L'Annonce faite à Marie, semblant lancer un appel au public² .

De fait, la naissance scénique de cette Annonce va constituer un double événement théâtral : créée à Paris en décembre 1912 au Théâtre de l'œEuvre par Lugné-Poe, elle sera aussitôt recréée en Allemagne en 1913 au théâtre d'avant-garde de Hellerau.

\section{La création française : un auteur exigeant}

2 La création française d'une pièce de Claudel se révéla un succès d'autant plus méritoire qu'il était inattendu, la plupart des critiques et Lugné-Poe lui-même redoutant le manque d'efficacité scénique d'un théâtre si littéraire. De plus, la participation de l'auteur à la préparation du spectacle - non seulement indirectement, par courrier ${ }^{3}$, mais concrètement, lors de deux visité à Paris - accrut cette crainte. Claudel n'était pourtant pas si étranger qu'il le prétendait aux exigences scéniques. Ainsi il conçoit fort bien que « le livre n'est pas la scène $»^{4}$ et, à cet effet, accepte volontiers de procéder à diverses coupures dans son texte, notamment à l'acte IV, si lyrique. Là où le dramaturge suscite l'inquiétude, c'est dans sa conception de la direction et de la gestuelle. En tant que poète, Claudel est particulièrement sensible à la musique, celle de ses vers et celle des voix qui les portent :

La division en vers que j'ai adoptée, fondée sur les reprises de la respiration et découpant pour ainsi dire la phrase en unités non pas logiques mais émotives, facilitera à mon avis l'étude de l'acteur. ${ }^{5}$

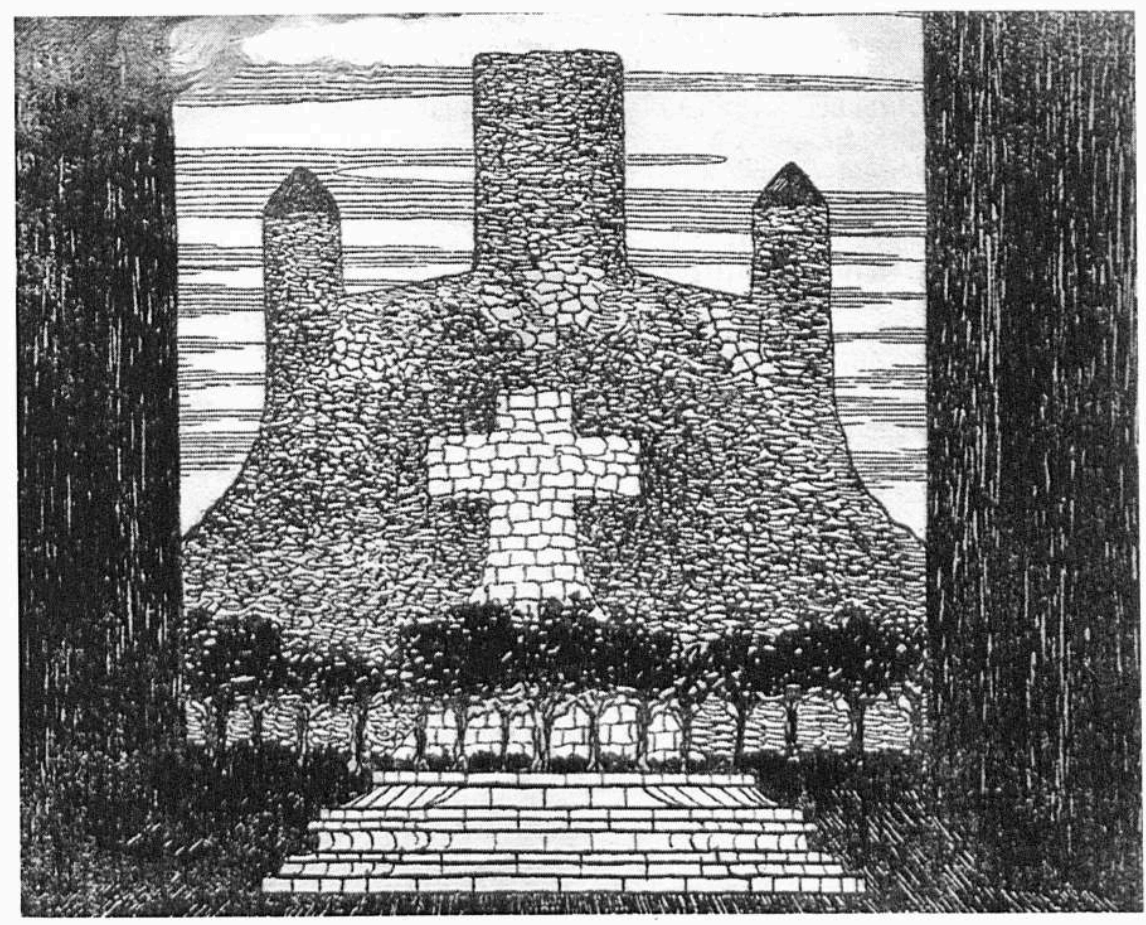

En défendant ainsi une déclamation émanant du rythme intérieur fondamental de la respiration, le poète-dramaturge rejette la récitation arythmique de la plupart des comédiens professionnels de l'époque. De même, sur le plan de la gestuelle, en 
recherchant le " mouvement qui se déploie vers son terme ${ }^{6}$, il propose une esthétique de la stylisation opposée aux gestes stéréotypés en usage. C'est pourquoi il préfère les jeunes acteurs, qui font primer la ferveur sur la technique. Cette opinion rejoint celle de Lugné-Poe qui, en tant que directeur du Théatre de l'ÆEuvre, propose plusieurs acteurs de sa troupe : lui-même jouera le Père, Anne Vercors; Roger Karl - à défaut d'Henri Rollan - sera Jacques Hury, et Victor Magnat Pierre de Craon. Une comédienne débutante, Marcelle Frappa, grande femme brune à l'aspect sauvage, correspondra bien à l'âpre Mara. Mais le choix de Violaine pose problème. Au départ, Claudel avait prévu Marie Kalff, si désireuse naguère de monter son théâtre, mais une maladie ne la rend plus disponible; Lugné-Poe propose alors Louise Lara, claudélienne convaincue et familière des théâtre d'art, mais sociétaire de la Comédie Française, et suscitant ainsi les craintes de l'auteur, qui doit cependant l'accepter.

\section{Jean Variot, un décorateur inventif}

4 Si la réflexion et l'activité scénique déployées par Claudel marquent considérablement l'interprétation des acteurs, elles demeurent étrangères aux préoccupations plus techniques, en particulier à l'aménagement du décor, qui n'apparait pas pour autant moins original. Ce domaine était de plus en plus négligé par Lugné-Poe lui-même, qui avait pourtant collaboré à ses débuts à l'Euvre avec les peintres nabis. C'est pourquoi le metteur en scène se laisse imposer par Claudel le décorateur Jean Variot. Ce dernier a étudié la scénographie en Allemagne, notamment dans la ville alors à l'avant-garde de la rénovation scénique, Munich, ou le décor se caractérise par un souci de simplification et de stylisation, afin de mettre en valeur le texte et les acteurs. C'est cette conception que Variot entend adapter à L'Annonce, assez audacieusement à une époque où au contraire en France la complication semble envahir la scène. Une telle esthétique de la sobriété, que le décorateur va jusqu'à qualifier d' «absence de décor ", semble d'ailleurs nécessaire au manque de moyens du Théâtre de l'œuvre, qui va stimuler l'ingéniosité : ainsi, à l'acte III, «Chevoche et la forêt seront figurés par de longues bandes de calicot brun, découpées et tombant du cintre (...) et l'abondance de ces fûts procurera une impression farouche de forêt assez impénétrable ${ }^{7}$. Pour l'éclairage, Variot innove en remplaçant rampes et herses par des projecteurs, mais ils demeurent trop rares et la scène reste souvent assez obscure. De plus, l'exiguïté de la salle Malakoff, où devra se jouer la pièce ${ }^{8}$, impose un dispositif réduit à deux parties : un proscenium habillé de tentures sombres et une arrière-scène présentant des toiles de fond.

Mais un somptueux cadre de scène enchâsse le spectacle "dans un écrin d'or et de cuir » où «le mystère de Paul Claudel déroulera ses rythmes et ses figures, telle une série merveilleuse d'image d'Épinal qui s'illumineraient, vivraient, chanteraient sous la baguette de quelque magicien $»^{9}$. Ainsi, tout en accentuant la convention théatrale en isolant la scène du monde extérieur, ce cadre crée une atmosphère sacrée; plus généralement, l'ensemble du décor a souvent été associé aux tableaux des primitifs italiens. Mais cette stylisation décorative de Variot se heurte à une exigence claudélienne de précision réaliste : à ce moment, le dramaturge veut illustrer son texte à la lettre, sans se rendre compte qu'un décor réaliste redoublera ce que le texte a déjà de concret. Variot se bornera à appliquer quelques éléments (une porte, une cheminée, une fontaine et surtout un bel arbre final) contre les toiles de fond, évitant ainsi un réalisme pesant ${ }^{10}$. En somme, les premiers décors de L'Annonce ont profondément 
innové dans la mesure où, à contre-courant des goûts de l'époque, ils ont révélé une esthétique de la sobriété et de la stylisation, au service de la vérité humaine ${ }^{11}$.

\section{Un succès inattendu}

5 Dans ces conditions, la création d'une pièce de Claudel, jouée dans une interprétation et des décors singuliers, semblait avoir tout pour dérouter le public. Or les trois représentations des 20, 22 et 23 décembre 1912, malgré la discrétion de leur annonce, furent un succès. La beauté poétique du texte allié à l'aspect sacré du spectacle, auxquels se joignirent l'intimité de la salle et la proximité de Noël, plongent les spectateurs - avertis pour la plupart, tels André Gide ou Jean Schlumberger - dans un pieux recueillement :

Le public le plus difficile du monde, le public léger, railleur, intelligent et sensible des "générales", a écouté avec émotion, avec admiration, l'œuvre lyrique, symbolique et mystique de $\mathrm{M}$. Claudel. Un silence frémissant, plus presque que l'applaudissement, s'étendait sur la salle et semblait vibrer. L'envie d'applaudir paraissait sans cesse et demeurait contenue. ${ }^{12}$

Manuscrit de Paul Claudel, revue de l'Éuvre

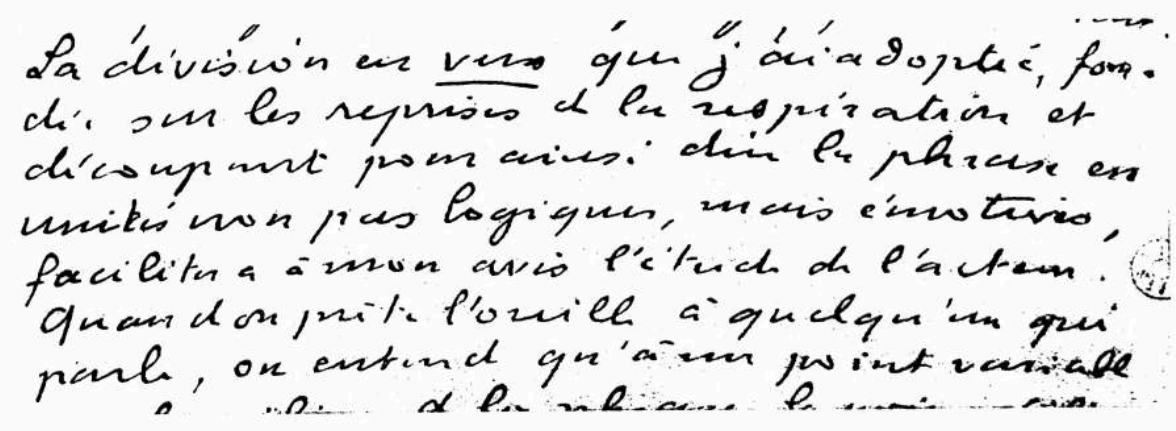

6 La critique, heureusement surprise, confirme l'émotion du public. Malgré quelques réticences à l'égard d'un aspect rhétorique du langage claudélien et d'une monotonie excessive de la diction, les journalistes louent surtout la ferveur de l'interprétation, en particulier chez Victor Magnat et Marcelle Frappa, les aspects plus proprement scéniques suscitant moins de commentaires. Mais Lugné-Poe et Variot n'avaient-ils pas précisément choisi la discrétion dans ces domaines pour mettre en valeur le texte ? On apprécie toutefois l'atmosphère mystérieuse, voire mystique, créée par les décors et l'éclairage, qu'accentue encore, sur le plan musical, les chœurs liturgiques de la Schola Cantorum. L'essentiel réside en tout cas dans la reconnaissance, contre toute attente, des vertus scéniques de L'Annonce, anticipant celles des autres pièces claudéliennes. "Le théâtre de Claudel est du théâtre, et du très bon théâtre ; il est fait pour être joué ", affirme Francis de Miomandre dans L'art moderne. Dès lors Lugné-Poe et Claudel luimême ne vont pas manquer d'exploiter aussitôt le succès de cette création. La mise en scène du Théâtre de l'ÆEuvre sera reprise brillamment à l'étranger au début de 1913 (à Bruxelles, Amsterdam et surtout Francfort, où l'auteur, Consul général dans cette ville, sera couronné sur scène), puis médiocrement en France : en mai à Paris, en décembre et au début de 1914 en province où la seule représentation marquante a lieu, le 26 mai à 
Lyon, lors d'une soirée organisée par Gaston Baty (qui montera la pièce en 1921 ) $^{13}$. La carrière de L'Annonce semble ainsi s'amorcer plus heureusement à l'étranger que dans son propre pays. C'est d'ailleurs en Allemagne, à Hellerau, qu'elle va connaître, en octobre 1913, une seconde naissance scénique.

\section{La reprise à Hellerau}

Cette mise en scène de Hellerau, étonnamment moderne pour l'époque, a recréé la pièce et a constitué un événement théâtral européen qui a enthousiasmé claudel.

L'Annonce avait été dès 1912 traduite en allemand, ou plutôt germanisée sous le titre Verkündigung, puis éditée en janvier 1913 par le fervent claudélien Jakob Hegner. Il sera également l'initiateur des représentations de Hellerau car, installé depuis peu dans ce lieu unique, il pense tout naturellement y présenter une pièce si originale. En 1913, Hellerau est un village de 2000 habitants situé à $5 \mathrm{~km}$ de Dresde, dont le site champêtre a séduit des architectes, des artisans et des artistes désireux d'y créer une cité-jardin de l'avenir. Pour réunir ces travailleurs, on vient d'y ouvrir un théâtre d'avant-garde, l'Institut d'Art : il abrite notamment les créations du musicien genevois Emile JacquesDalcroze, mais veut représenter du théâtre, et Verkündigung va ainsi inaugurer la Société Dramatique de Hellerau. Lorsque Claudel découvre ce lieu en février 1913, il est enthousiasmé par l'aspect révolutionnaire de la salle de spectacle, inspirée des conceptions d'Adolphe Appia : dans ce grand rectangle sans cadre de scène ni rideau, on peut modeler librement le sol scénique et la lumière irradie en l'absence d'un foyer visible ; le dramaturge remarque aussitôt la liberté que permet un tel aménagement ${ }^{14}$. La préparation de Verkündigung est alors confiée à des membres de la Société Dramatique - Emil Strauss pour la mise en scène, Wolf Dohrn pour l'organisation matérielle, et Alexander von Salzmann pour l'éclairage - qui travaillent pendant que Claudel est à Francfort. Mais lorsqu'à la fin de juin ce dernier revient à Hellerau, il est si enthousiasmé par une représentation de l'Orphée de Gluck, monté par Jacques-Dalcroze dans un décor architectural de praticables, plans inclinés et escaliers, qu'il décide aussitôt que son Annonce ne peut être présentée autrement. Il conteste alors la mise en scène préparée jusque là, insuffisamment adaptée selon lui aux possibilités de l'espace scénique, et n'hésite pas à remercier le metteur en scène Emil Strauss pour se substituer à lui ${ }^{15}$. Il amorce immédiatement, au début de juillet, une nouvelle mise en scène, puis laisse Dohrn et Salzmann s'en occuper, avant de revenir la diriger en septembre en assistant aux répétitions.

\section{Une mise en scène signée Claudel}

9 Cette nouvelle mise en scène se caractérise par une originalité qui apparaît d'abord dans la nature du dispositif scénique et l'utilisation de la lumière, avant de se manifester dans le travail du texte. C'est pourtant ce dernier aspect qui avait retenu l'auteur lors de la préparation de la création parisienne, mais ce qui le passionne désormais, c'est d'expérimenter des moyens techniques d'avant-garde :

On s'est servi exclusivement, pour la mise en scène de L'Annonce, de ces ressources naturelles: lumière et architecture. Pas d'accessoires, pas de peinture pas de cartonnages. Aucune recherche $\mathrm{du}$ pittoresque. Tout est subordonné à l'interprétation dramatique. La scène n'a pour but que de fournir d'avance à l'action son armature et la construction organique de plans et d'élévations suivant lesquels elle doit se développer ${ }^{16}$. 


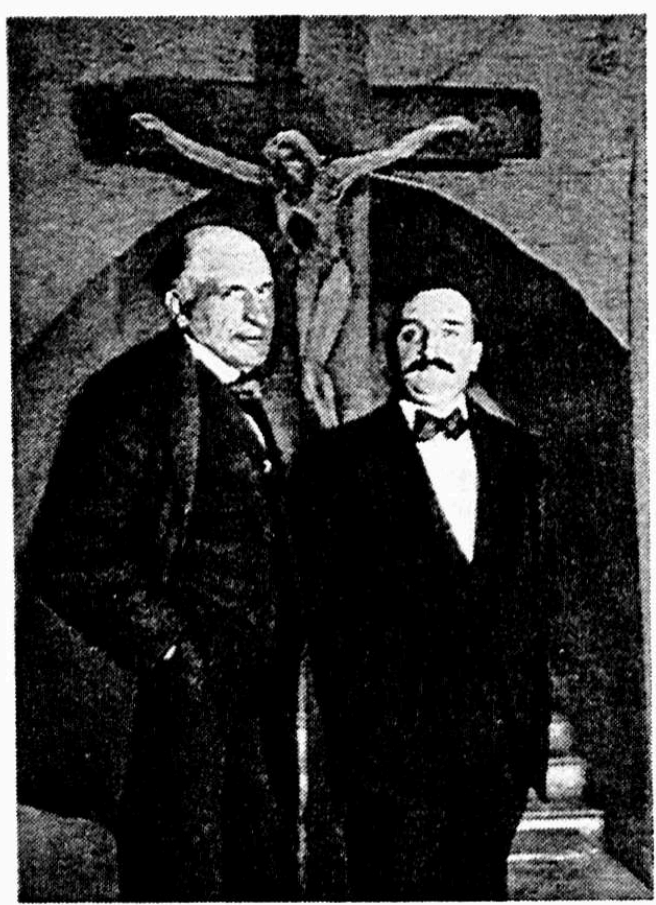

10 Ces derniers mots font allusion à l'une des grandes innovations de la mise en scène de Hellerau, le dispositif à étages, admiré par Claudel dans Orphée, qui se répartit en trois niveaux superposés reliés par des escaliers. Au niveau inférieur, un renforcement évoque à la fois l'âtre et la caverne de Violaine à l'acte III ; au niveau supérieur se trouve la porte ouverte sur le monde spirituel ; entre ces deux niveaux extrêmes, un étage intermédiaire correspond à des moments décisifs de l'action, comme la grande scène entre Violaine et Jacques à l'acte II. Plutôt que d'impliquer un ordre hiérarchique comme dans la scène à mystères médiévale, les étages reliés présentent les différentes portées des événements : Claudel veut mettre en valeur "le passage " qui assure la continuité des scènes, de sorte que l'action évolue sans jamais se briser ${ }^{17}$. Au-delà de ce goût pour la simultanéité scénique, l'auteur traduit dans la scène à étages une intention métaphysique, qui n'existait pas dans le dispositif d'Orphée, en appliquant la théorie des « acteurs permanents ». Le mot " acteur » signifie ici tout ce qui agit, intervient, " est là ", mais en tant qu'objets inertes, comme le feu, la table, la porte. Chacun de ces éléments fait fonction de "foyer ", suggère un lieu dont il propage les attributs ; ainsi les objets, loin de n'être qu'une vaine reproduction du réel, signifient quelque chose : «Ce qui était le foyer devient la flamme toujours vigilante, ce qui était la table devient l'autel, ce qui était la porte devient la porte du ciel ${ }^{18}$. Cette conception permet dès lors d'échapper à la succession linéaire habituelle: grâce à la présence simultanée des " acteurs permanents ", l'espace scénique porte déjà la scène à venir tout en restant marqué par la précédente, et cette union de plusieurs moments engendre une sorte de présent universel. Ainsi Claudel est aux antipodes du réalisme qu'il revendiquait pourtant lors de la création parisienne en exigeant de Lugné-Poe un respect scrupuleux de ses indications scéniques. Au contraire, à Hellerau, le décor devient une sorte d'épure ne se prêtant à la présence d'aucun détail concret : 
Les décors stylisés au maximum étaient faits de larges cubes architecturaux recouverts d'étoffe bleue, avec lesquels on pouvait construire des marches et constituer des paliers de différentes hauteurs (...). Des bandes d'étoffe bleue descendaient du plafond et suggéraient, selon qu'elles étaient rapprochées ou éloignées, des arbres, des maisons, un mur etc ${ }^{19}$.

11 Cet extrême dépouillement du décor se trouve largement compensé par la subtilité des éclairages. Le dispositif technique conçu par Salzmann permet à la lumière de modeler l'espace et de sculpter les corps des acteurs en créant « une espèce d'ambiance laiteuse, d'atmosphère élyséenne, qui rend à la troisième dimension son honneur méprisé et fait de tout corps une statue $»^{20}$. Mais Claudel entend également attribuer à la lumière une signification symbolique ; elle accentue la dimension spirituelle de la pièce en donnant à l'action terrestre son couronnement surnaturel : cette lumière, c'est l'esprit, la grâce, intervenant dans les scènes essentielles sous la forme d'une ogive lumineuse.

L'architecture scénique et la lumière caractérisent donc les deux fondements essentiels de l'originalité de la mise en scène claudélienne de L'Annonce à Hellerau, mais le dramaturge n'en oublie pas pour autant l'interprétation des acteurs, à laquelle il s'était intéressé au Théâtre de l'œuuvre. A cet égard, des renseignements nous sont donnés par le Regiebuch de Hellerau, exemplaire de la deuxième édition de Verkündigung en 1913, annoté par Claudel, Hegner et Dohrn ${ }^{21}$. Ce document révèle que Claudel, peut-être à cause de l'extrême stylisation de sa mise en scène effaçant tout réalisme psychologique, a pris quelque distance avec ses personnages, notamment avec Violaine : en effet, il indique souvent que son héroïne doit jouer sur un ton ironique à l'égard de sa sœur, de sa mère et de Jacques Hury. Mais de nombreuses autres annotations confirment le désir claudélien d'une diction musicale, déjà recommandée aux acteurs de la création parisienne : le dramaturge a souvent inscrit un signe codé (une double croix) devant les passages qu'il estime spécialement musicaux. Cette musicalité doit se trouver en harmonie avec la stylisation des gestes traduisant un rythme intérieur, véritable rituel propre à une sorte de célébration mystique.

\section{Un retentissement mitigé}

Une mise en scène si nouvelle n'allait pas manquer d'avoir un grand retentissement. Contrairement à la discrétion de la création parisienne, les représentations de Hellerau revêtent beaucoup de prestige et ont été considérées comme "une des dernières grandes manifestations culturelles internationales avant la Première Guerre ${ }^{22}$. La première du 5 octobre - deux autre représentations suivront le 11 et le 19-apparait comme une longue célébration à laquelle assistent d'éminents représentants du monde politique et artistique ${ }^{23}$. Mais tout ce beau monde, souvent venu pour l'auteur plus que pour sa pièce, semble avoir eu des avis partagés sur le spectacle. Les critiques, eux, n'apprécient guère les comédiens à l'exception de Mary Dietrich, actrice berlinoise du grand metteur en scène Max Reinhardt, qui incarne Mara. Surtout, ils manifestent une certaine réserve devant la mise en scène qui, malgré sa beauté plastique, laisse une impression de sévérité, de sécheresse, voire d'artifice. Même les savants éclairages de Salzmann paraissent artificiels: effectivement, il n'était peut-être pas judicieux de plonger les paysans de L'Annonce dans le bain lumineux qui avait été conçu pour les âmes bienheureuses d'Orphée. Là semble résider la faille de ce spectacle dépouillé : en cédant impulsivement à son enthousiasme, Claudel n'a-t-il pas voulu trop systématiquement appliquer à sa pièce une mise en scène qui occultait son aspect 
concret, charnel ${ }^{24}$ Cependant un tel spectacle a su révéler, grâce à une nouvelle esthétique scénique, la profonde dimension intérieure de L'Annonce, qui ne sera peutêtre jamais autant « célébrée » qu'à Hellerau ${ }^{25}$.

\section{Une existence scénique qui s'impose}

13 Ainsi, les deux premières mises en scène de L'Annonce, si elles comportent des imperfections, auront prouvé que cette pièce - et au-delà, les autres pièces de Claudel était bien faite pour être représentée, contrairement aux opinions les plus répandues auparavant: L'Annonce connaîtra même l'une des plus impressionnantes carrières scéniques du XXe siècle. Plus précisément, en se fondant sur de nouvelles conceptions esthétiques, ces deux créations ont orienté l'adaptation scénique du drame de Violaine dans le sens d'une stylisation et d'une sobriété, peut-être trop naïve à Paris et trop sèche à Hellerau, mais permettant d'aller au plus profond, de comprendre le pouvoir du mystère et de la foi, sans se perdre dans les futiles apparences du réalisme. Ces deux spectacles ont également révélé l'engagement de Claudel dans les mises en scène de sa pièce : encore timide au Théâtre de l'Euvre, il s'est vite imposé à Hellerau, et d'une manière générale le dramaturge aimera toujours participer à l'enfantement scénique de ses œuvres. Enfin, la double naissance de L'Annonce sur les planches, en ayant su faire exister le théâtre claudélien, a inauguré les mises en scène des autres pièces de l'auteur: L'Echange, puis L'Otage suivront en 1914, et lentement mais irréfutablement, le théâtre claudélien s'imposera comme l'un des plus importants du siècle.

\section{NOTES}

1. Les intertitres sont de la rédaction.

2. Sur ces remaniements scéniques, voir Alain Béretta, La vie scénique d'une pièce de théâtre: L'Annonce faite à Marie de Paul Claudel (1912-1995), Thèse de Doctorat, Université de Nancy II, 1996, p.13-54.

3. Claudel est alors Consul général à Francfort. Sa correspondance avec Lugné-Poe est publiée dans Cahiers de Paul Claudel 5, Gallimard 1964. Il faut lire aussi les souvenirs de Lugné-Poe, Dernière pirouette, Editions du Sagittaire, 1946.

4. Claudel à Lugné-Poe, 27 Août 1912, Cahiers de Paul Claudel 5, p.81.

5. Claudel, Mes Idées sur la manière générale de jouer mes drames, op.cit., p.185.

6. Ibid., p.186.

7. Lugné-Poe, Dernière Pirouette, p.47-8. Voir l'illustration de ces arbres de Chevoche dans Denis Bablet, Le Décor de théâtre de 1870 à 1914, Editions du CNRS, 1965, planche nº 168 .

8. Ne possédant pas de lieu fixe, le Théâtre de l'œuvre n'a pu trouver que cette petite salle, située au 56bis de la rue Malakoff ( $16^{\mathrm{e}}$ arrondissement, et habituellement plus réservée à des séances de patronage qu'à du théâtre.

9. Robert Brunelles, « Décors nouveaux », Gil Blas, 17 décembre 1912. 
10. Pour plus de précisions sur ces décors, voir Jean Variot, «Quelques considérations sur la mise en scène de L'Annonce faite à Marie » et "Les décors de L'Annonce faite à Marie », Bulletin de l'Euvre, octobre-novembre 1912, textes repris dans Euvres complètes 9 de Claudel, p.259-68.

11. Ces décors «marquent une date dans l'histoire du théâtre français » selon L'Encyclopédie du théâtre contemporain, tome I, collection Théâtre de France, 1957, p.58.

12. Henry Bidou, Le Journal des débats, 23 décembre 1912.

13. Quoi qu'il en soit, Lugné-Poe, heureux et fier d'avoir, le premier, créé une pièce de Claudel, restera fidèle à L'Annonce jusqu'à la fin de sa vie théâtrale : il montera la pièce une dernière fois en 1927, à la Maison de l'CEuvre, dans une mise en scène très proche de celle de 1912.

14. Cette salle décrite par Claudel lui-même dans deux articles anonymes: "Sur le théâtre d'Hellerau » et "L'Annonce faite à Marie de Paul Claudel au théâtre allemand d'Hellerau », 1913, textes repris dans Mes idées sur le théâtre, Gallimard, 1966, p.40-5.

15. Les relations conflictuelles entre Claudel et les membres de la Société Dramatique de Hellerau sont évoquées dans les Archives Martin Buber conservées à la bibliothèque nationale et universitaire de Jérusalem. Buber était président de la Société.

16. "L'Annonce faite à Marie de Paul Claudel au théâtre allemand d'Hellerau ", Mes idées sur le théâtre, p.44.

17. Dans ce même article, p.45, Claudel donne plusieurs exemples de cette continuité de l'action aux différents étages.

18. Ibid., p.44.

19. Darius Milhaud, Notes sans musiques, Julliard, 1949, p.64.

20. «Sur le théâtre d'Hellerau ", Mes idées sur le théâtre, p.42.

21. Ce Regiebuch a été découvert en 1994 dans les archives du compositeur autrichien Egon Wellesz, qui semble avoir pensé faire un opéra à partir de L'Annonce. Il est actuellement consultable aux Archives de la Société Paul Claudel à Paris.

22. Dominique Bourel, « Sur Claudel et l'Allemagne », Question de, n94, novembre 1993, p.191

23. Voir Edwin Maria Landau, Paul Claudel auf deutschsprachigen Bühnen, Prestel Verlag, Munich, 1986, p.44.

24. C'est en tout cas l'opinion de Jacques-Dalcroze et d'Adolphe Appia: voir l'ouvrage collectif Emile Jacques-Dalcroze, l'homme, le compositeur, le créateur de la rythmique, Editions La Baconnière, Neuchâtel, 1965, p.448.

25. Cette mise en scène restera certainement pour Claudel le meilleur souvenir scénique de sa pièce. En 1933, alors qu'elle a déjà été montée dans de nombreux endroits, il avoue à son traducteur italien, le professeur Casnati: «Ce qui a été fait à propos des mises en scènes de L'Annonce m'a toujours paru médiocre, sauf peut-être à Hellerau », Archives de la Société Paul Claudel.

\section{AUTEURS}

\section{ALAIN BERETTA}

Docteur ès lettres 